\title{
The Average Mutual Information Profile as a Genomic Signature Mark Bauer ${ }^{1}$, Sheldon M Schuster ${ }^{2}$ and Khalid Sayood*1
}

\author{
Address: ${ }^{1}$ Department of Electrical Engineering, University of Nebraska, Lincoln, NE USA and ${ }^{2}$ Keck Graduate Institute, Claremont Colleges, \\ Claremont, CA, USA \\ Email: Mark Bauer - mark@engr.unl.edu; Sheldon M Schuster - Sheldon_Schuster@kgi.edu; Khalid Sayood* - ksayood@eecomm.unl.edu \\ * Corresponding author
}

Published: 25 January 2008

BMC Bioinformatics 2008, 9:48 doi:10.1/86/|47|-2105-9-48

This article is available from: http://www.biomedcentral.com/I47I-2105/9/48

(c) 2008 Bauer et al; licensee BioMed Central Ltd.

This is an Open Access article distributed under the terms of the Creative Commons Attribution License (http://creativecommons.org/licenses/by/2.0), which permits unrestricted use, distribution, and reproduction in any medium, provided the original work is properly cited.
Received: 4 July 2007

Accepted: 25 January 2008

\begin{abstract}
Background: Occult organizational structures in DNA sequences may hold the key to understanding functional and evolutionary aspects of the DNA molecule. Such structures can also provide the means for identifying and discriminating organisms using genomic data. Species specific genomic signatures are useful in a variety of contexts such as evolutionary analysis, assembly and classification of genomic sequences from large uncultivated microbial communities and a rapid identification system in health hazard situations.
\end{abstract}

Results: We have analyzed genomic sequences of eukaryotic and prokaryotic chromosomes as well as various subtypes of viruses using an information theoretic framework. We confirm the existence of a species specific average mutual information (AMI) profile. We use these profiles to define a very simple, computationally efficient, alignment free, distance measure that reflects the evolutionary relationships between genomic sequences. We use this distance measure to classify chromosomes according to species of origin, to separate and cluster subtypes of the HIV-I virus, and classify DNA fragments to species of origin.

Conclusion: AMI profiles of DNA sequences prove to be species specific and easy to compute. The structure of AMI profiles are conserved, even in short subsequences of a species' genome, rendering a pervasive signature. This signature can be used to classify relatively short DNA fragments to species of origin.

\section{Background}

The existence of patterns that can be used as a signature of data is indicative of statistical or deterministic structures in the data. In DNA sequences this structure can be due to biological processes which involve the DNA or they may appear because of events and processes in the evolutionary history of the DNA. There have been significant efforts in understanding the sequential structure and complexity of DNA using various approaches, information theoretic measures or other mathematical models.
The standard approach to studying statistical relationships in a sequence is the use of correlation profiles or spectral profiles such as periodograms and power spectrums. To translate the sequence of letters that form the DNA sequence into a sequence of numbers, which can then be easily analyzed using autocorrelation or spectral techniques, different mappings have been proposed by Gates [1], Voss [2] and Peng et al. [3]. The power spectral densities obtained from these approaches show a power law relationship, which points to the existence of long 
range correlations. A number of models have been proposed to account for these long range correlations $[4,5]$.

Somewhat distinct from statistical models, several researchers have used information theoretic measures to study the characteristics of the DNA sequence. A description of the use of information measures to study DNA sequences can be found in Gatlin [6] and Roman-Roldan et al. [7]. Schneider et al. [8-10] have used information theoretic measures in a number of interesting ways from studying the information content at nucleotide binding sites to expediting alignment. However, most of the applications of information theory has been to the study of the correlation properties of DNA sequences [7,11-14]. A significant portion of these are directed toward obtaining a mechanism for the long range correlation properties of the DNA sequence, while others study the ability of information theoretic measures to differentiate between coding and non-coding regions or to demonstrate a close relationship between sequence compositional complexity of the DNA sequence and the biological complexity of the organism to which the sequence belongs $[15,16]$.

Another line of approach to understand the compositional structure of DNA sequences has focused on frequency profiles of short oligonucleotides. Karlin and coworkers [17-19] have shown that there is a compositional bias in bacterial genomic sequences. Blaisdell and coworkers have shown the same for viral sequences [20]. Karlin et al. [21] have used this compositional bias in bacterial genomes to infer evolutionary relationships. The compositional biases of DNA sequences have also been studied from the point of view of linguistics. Brendel et al. $[22,23]$ provide a technique to identify possible short oligonucleotide sequences within DNA sequences based on the deviation of the frequency of occurrence of these sequences from their expected value. Bultrini et al. [24] propose the existence of a pentamer vocabulary characterizing intron and intron-like intergenic tracts. This approach has been used for intron/exon discrimination as well as for gene finding.

One important implication of different approaches to characterize the structure and complexity of DNA sequences has been the interest in discovering patterns in genomic sequences that can be used as signatures of species. Such signatures can be useful in a wide variety of contexts. If differences between signatures can be related to evolutionary distance they can be used for developing phylogenetic relationships and for understanding evolutionary processes $[18,20,21,25]$.

The existence of reliable genomic species signatures would have significant implications in developing a rapid identification system using DNA sequences. Bacterially trans- mitted diseases continue to be a major threat to health with increasing threat from previously unknown variants, which have antibiotic resistance. The threat of bioterrorism adds to this potentially lethal mix. In order to respond to a disease outbreak, whether initiated by natural or artificial means, there is an urgent need for rapid identification of infectious agents to limit exposure and initiate treatment. Therefore, it is important to identify and understand structures within the genome of organisms which differentiate them from each other and from more benign organisms.

The recent presentation of the genomic sequences of large microbial populations presents yet another application for a species signature $[26,27]$. Tyson et al. used random shotgun sequencing of DNA from a natural acidophilic biofilm to identify the structure of the uncultivated microbial community [26]. In a similar approach Venter et al. targeted a much more complex microbial population collected from the Sargasso Sea region [27]. In this latter study, approximately 3 million reads yielding about 1.6 billion base pairs of DNA sequences were generated. It is believed that these sequences belong to at least 1,800 genomic species. These approaches present a very complicated problem of identification and assembling shotgun reads coming from an unknown number of species. Signatures which can be used to identify and distinguish between fragments based on their species of origin would be useful in this process.

Most existing approaches to defining species specific signatures are based on frequency distribution of oligonucleotides, also referred to as "words" [28-30]. However, the choice of the length of the words and the DNA sequence window in which the frequency profiles of the words are observed not only result in data explosion but also change the composition of the resulting signature. In this paper we present AMI profile of DNA sequences as a candidate for species signature. AMI profiles are pervasive in the sense that they can be detected in small fragments of the DNA sequence. The proposed genomic signature is a vector where the $k^{\text {th }}$ entry is the AMI between nucleotides that are $k$ locations apart. AMI profiles are generated virtually free form any parameters resulting in an automated unbiased calculation. We also use this signature to develop a simple, computationally inexpensive measure of distance between genomic sequences. We validate this distance measure by using it with standard phylogenetic algorithms to perform unsupervised clustering.

AMI was first introduced for studying the communication of signals under noisy channel conditions [31]. In communication theory it is interpreted as a measure of the information contained in one event $X$ about another event $Y$ (or vice versa). In the bioinformatics area the aver- 
age mutual information has been used to detect correlated mutations at noncontiguous sites in a sequence [13], for secondary structure prediction $[32,33]$ to investigate correlations between sites in protein sequences $[7,11,12]$, and to differentiate between coding and noncoding regions [34]. Slonim et al. [35] use average mutual information to formulate the clustering problem in a variety of settings including gene expression, stock prices, and movie ratings. Slonim et al. [36] also use average mutual information to study the relationships between genes and their phenotypes.

Berryman et al. [37] have used the average mutual information profile to demonstrate long-range correlation in DNA sequences. More important, from the perspective of this work, they show that the long-range structures evident in the profile of a sequence results from evolutionary events such as additions, deletions, and insertions of repetitive elements. This view is further validated by the work of Holste et al. [38] which focuses on two specific peaks at $k=135$ and $k=160$ in the average mutual information profile of Human Chromosomes 20, 21, and 22. When they replace Alu repeats in the chromosomes with random sequences these peaks disappear validating their contention that the peaks occur due to the presence of Alu repeats. The discrimination property of AMI was also demonstrated by Dehnert et al. [39,40] for eukaryotic chromosomes. Dehnert et al. [39] use the Euclidean distance between AMI profiles and coefficients of autoregressive models to discriminate between various eukaryotic genomes. Hummel et al. [41] use average mutual information to analyze protein sequence motifs. In the work of Hmmel et al., as in earlier works [13,42] the different sequences are first aligned using a multiple sequence alignment and treated as realizations of a random process. The probabilities needed to compute the average mutual information are then obtained from this ensemble.

These results indicate that on some level the AMI profile can be viewed as a representation of the evolutionary history of the organism. As the AMI profile is an average measure the structure evinced by the profile is likely to be pervasive. That is, this history should be reflected to some extent in all parts of the genome and sufficiently long fragments of the genome should have similar profiles. Organisms that are evolutionarily related have an extensive common history. If the AMI profile reflects evolutionary history, this common history should be reflected in similarity of their AMI profiles. In the following we present evidence to support this hypothesis, based upon which we suggest that the AMI profile is an excellent candidate for a species signature.

\section{Results}

\section{The AMI profile of chromosomes}

We begin with the largest fragments of available DNA sequences, the chromosomes of eukaryotes. Consider the AMI profile shown in Figure 1 corresponding to Human chromosome 1 . The abscissa corresponds to the distance between two bases in the sequence, while the ordinate is the value of the average mutual information. A larger value of the average mutual information for a particular value of $k$ corresponds to higher dependence between bases $k$ apart. Clearly we would expect higher dependence between bases closer than between bases further apart. The various peaks may be the result of a number of factors including the ratio of coding to noncoding regions and the existence of various kinds of repeats.

If we now plot the AMI profile for different chromosomes as shown in Figure 2a, we see that the peaks and valleys occur at identical locations. This is true of all chromosomes in spite of the significant differences in size and gene content. We have plotted the AMI profile for values of $k$ between 5 and 50 to better show the similarities. The same holds true for other values of $k$. Note that we have not tried to align the chromosomes which, given their diversity, would not have been feasible. Plotting the same chromosomes for mouse (mus musculus) in Figure 2b, we see again the similarity between the AMI profiles for the various chromosomes. We can also see that these profiles are distinct from those of the human chromosomes.

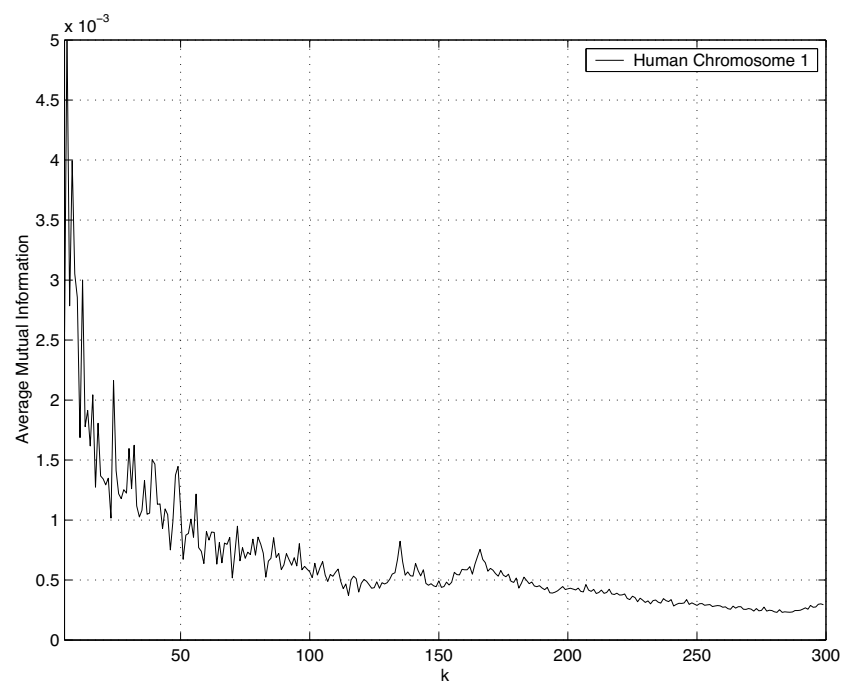

Figure I

Average Mutual Information Profile for Human Chromosome I plotted for $k \geq 5$. The $x$-axis is the distance between bases while the $y$-axis is the value of the average mutual information $I_{k}$. 


\section{a) Human Chromosomes}
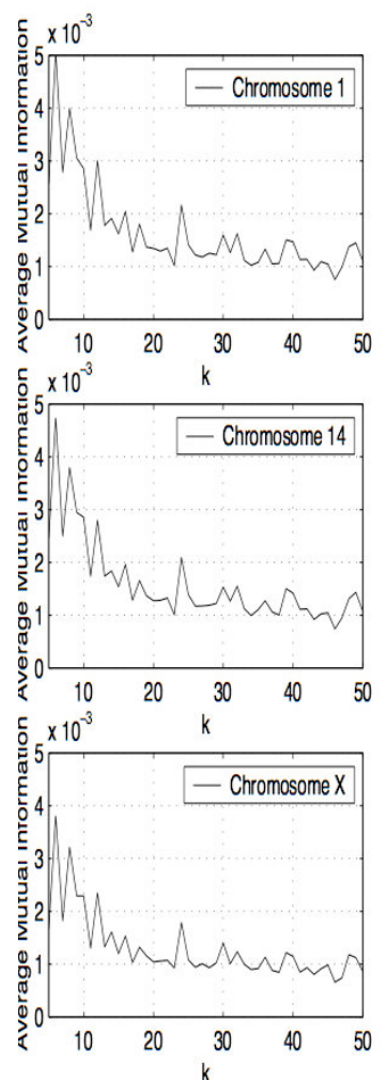
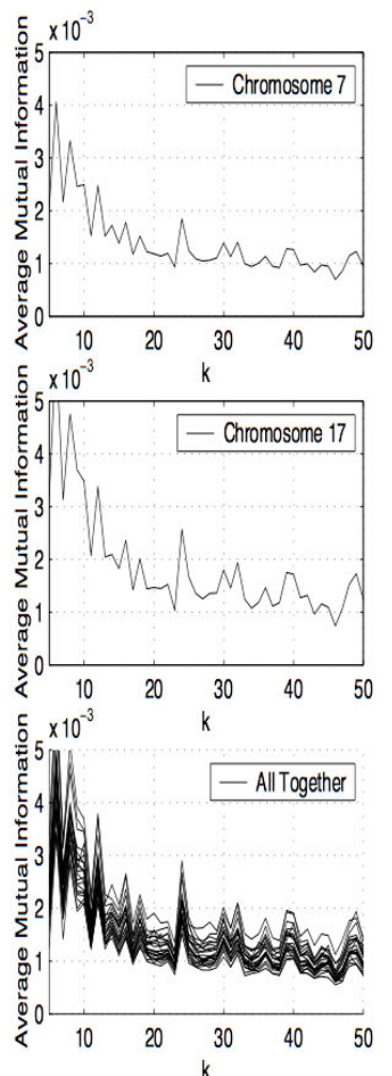

\section{b) Mouse Chromosomes}
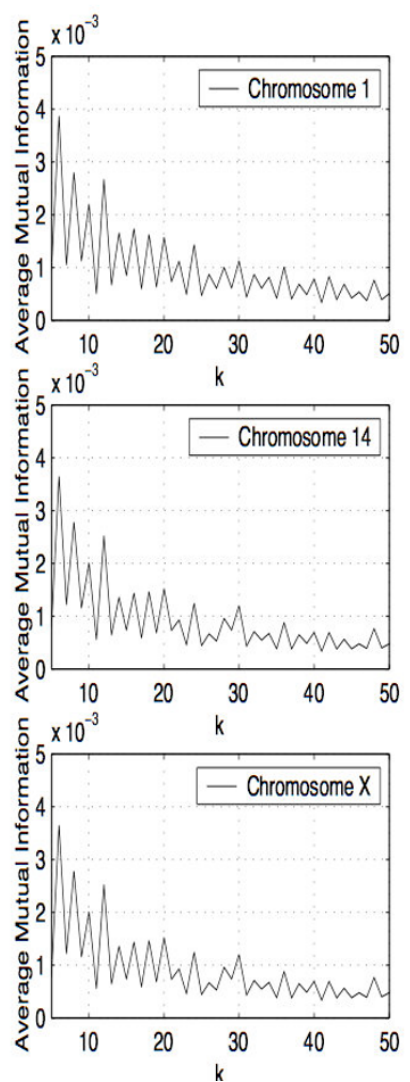
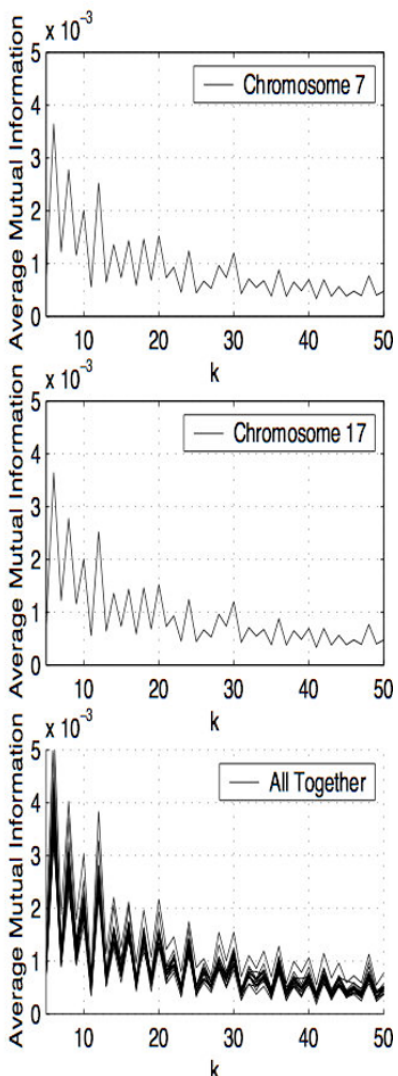

Figure 2

Average Mutual Information Profile for the Human Chromosomes plotted for values of $k$ between 5 and 50 , b) Average Mutual Information Profile for the Mouse Chromosomes plotted for values of $k$ between 5 and 50 .

If we repeat this experiment for the chromosomes of $C$. elegans we get the same result. Again, when we plot the profile we see a pattern of peaks and valleys which occur at the identical locations for all chromosomes of C. elegans. We demonstrate this with five chromosomes of $C$. elegans in Figure 3a. Again, while the pattern of peaks and valleys in the AMI profile is the same for all chromosomes of C. elegans, this pattern is distinctly different from the pattern of peaks and valleys in the human and mouse AMI profiles.

Finally we repeat the experiment for Saccharomyces cerevisiae. The results are shown in Figure $3 \mathrm{~b}$ (note the peaks at multiples of three reflecting a larger proportion of coding regions compared to the previous examples). Once more we obtain a sequence of peaks and valleys in the AMI profile which are the same for all chromosomes of $S$. cerevisiae, and this pattern of peaks and valleys is different from the patterns in the profiles of the other species.
We then plot AMI profiles for the complete E. coli sequence (accession number NC_000913) and a 0.5\% fragment of the sequence in Figure 4 to check for pervasiveness. The striking similarity between the profile suggests that AMI profiles can be used to identify random fragments of a DNA sequence with their species of origin.

We test this hypothesis by computing the correlation coefficient of the AMI profile of 100,000 $5 \mathrm{~kb}$ long fragments of the E. coli genome with the AMI profile of the entire sequence. We also compute the correlation coefficient of 100,000 random fragments from the $S$. aureus genome (accession number NC_002758) with the AMI profile of the E. coli genome. The histograms of the correlation coefficient are shown in Figure 5. The results clearly demonstrate both the pervasiveness of the AMI signature as well as its specificity.

Finally, to investigate the length of fragment required to compute a genomic signature we plot the average correla- 
tion of profiles of 1000 fragments of genomic DNA with a reference profile obtained from the entire genome in Figure 6. The size of the fragments are varied from 200 nucleotides to 10,000 nucleotides. In these experiments we have restricted the size of the AMI profile to sixteen in order to easily compute the profiles of short segments. The reference AMI profile is that of $\mathrm{E}$. coli and the fragments are from E. coli and S. aureus. As was to be expected, the plot shows that the correlation between the profiles of the fragments and the reference profile of the genome increases with increasing fragment length. While this is true for profiles of both E. coli and S. aureus fragments, the profiles of the E. coli fragments are consistently more correlated with the reference profile than the profiles of the $\mathrm{S}$. aureus fragments. This is true for all fragment sizes. This suggests that the AMI profile could be useful in classifying relatively short fragments. All these figures indicate the existence of a profile specific to a species. Using this as our motivation we develop a distance measure which can be used to classify genomic sequences to species of origin. We verify the utility of this metric by classifying retroviruses based on their host species and by classifying subtypes of the HIV-1 virus.

\section{A distance measure}

Noting that genomic sequences from the same species have similar pattern of peaks and valleys a numerical measure of the closeness of their AMI profiles can be obtained by looking at the correlation coefficient between the AMI profiles. As the larger values of the AMI profile for small values of $\mathrm{k}$ tend to mask the differences between AMI profiles we evaluate the correlation coefficient for values of $\mathrm{k}$ greater than 5 . In our simulations the upper limit for $\mathrm{k}$ was 512 . Using values of $\mathrm{k}$ greater than $512 \mathrm{did}$ not effect the results. We define the distance $d_{i j}$ between AMI profiles of the $i^{\text {th }}$ and $j^{\text {th }}$ sequences to be one minus the correlation coefficient. Note that to compute distances between sets of sequences we do not need to align these sequences. This is especially useful when we look at distances between chromosomes as multiple sequence alignment for whole genomes or chromosomes is an unsolved problem. The availability of an alignment-free approach to finding the distance between genomic sequences may considerably simplify the investigation of genomic relatedness of species based on their sequence information.

We apply this distance measure to three chromosomes from four species. The particular chromosomes are listed in Table 1. The distances between these chromosomes are shown in Table 2. Clearly, the distances between chromosomes from the same species are substantially smaller than the distances between chromosomes of different species. Furthermore the distances between the AMI profile of chromosomes of more closely related species such as mouse and human is substantially less than the distance between less closely related species such as mouse and yeast. For the species for which we have sequences available the pattern holds for other chromosomes as well.

It is difficult to show the data for all the chromosomes in tabular form. We have developed a visualization program (described in Methods), which gives a visual representation of the distances between AMI profiles. One representation of the distances of the chromosomes of the four species used in this experiment is shown in Figure 7. Note that as we are projecting from a multi-dimensional space into a two-dimensional space the representation is not unique. However, in order to show that a population can be separated into different classes all we need to show is clustering in a single representation. That all the genomic sequences can be assigned to their particular species is clear from the figure. The program provides a means of visualizing the distances between AMI profiles and qualitative evidence for clustering. We can also show visual evidence of clustering using a singular value decomposition. In the next section we show that these distances can be used in a quantitative manner with the UPGMA algorithm to provide unsupervised clustering.

\section{Grouping HIV subtypes}

The Human immunodeficiency viruses (HIV) represent a group of retroviruses that are distinct from endogenous retroviruses and are not presumed to have originated from human cellular DNA sequences. However, the life cycle of these viruses and their genome are essentially the same as that of all other retroviruses - reverse transcription of the RNA genome into proviral DNA followed by integration into host cell chromosomal DNA and the formation of progeny viral RNA genome by transcription from the proviral DNA. Of the two major types of the HIV virus, HIV1 is the more virulent and is the predominant strain. There are multiple subtypes of the HIV-1 virus with some degree of geographic segregation between the various subtypes. This geographic segregation argues for evolutionary differences between the different subtypes. As such, it should be feasible to differentiate between the different subtypes using the AMI profile. The results of our analysis of AMI profiles of the genomes of twenty one independent viral isolates listed in Table 3 are shown in Figure 8. The clustering approach used is described in the Methods section. We also show clustering by plotting three coefficients from the singular value decomposition of the AMI profiles in Figure 9. The UPGMA tree, constructed using the distance measure described earlier, corresponding to these isolates is shown in Figure 10.

The distance between members of each subgroup is relatively high as compared to DNAs of different chromosomes of the same species. However, the distance between AMI profiles from different subgroups is higher than 


\section{a) C. Elegans Chromosomes}
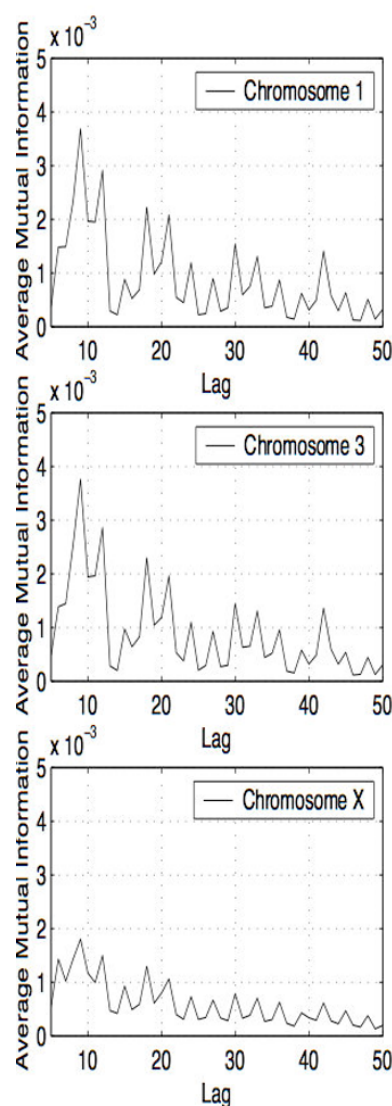
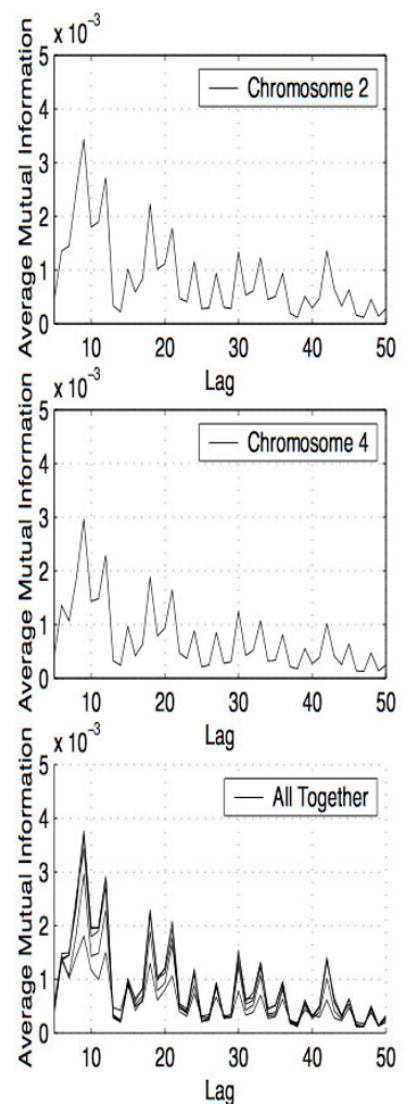

\section{b) S. Cerevisae Chromosomes}
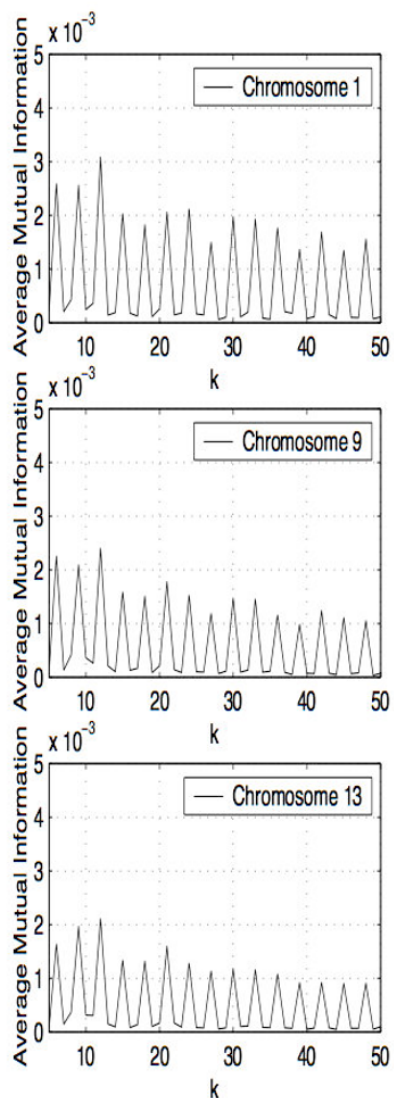
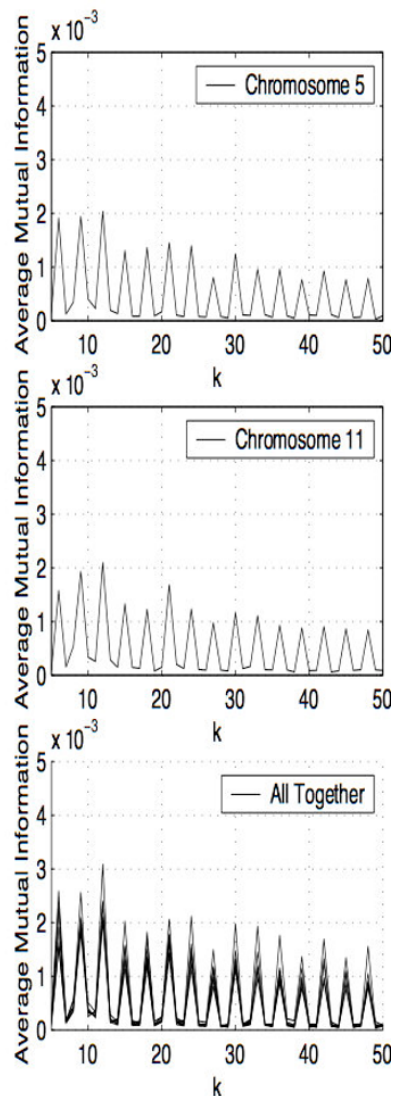

Figure 3

Average Mutual Information Profile for the C. elegans Chromosomes plotted for values of $k$ between 5 and 50 , b) Average Mutual Information Profile for the S. cerevisiae Chromosomes plotted for values of $k$ between 5 and 50 .

between members of the same subgroup. This is clear from the clustering evident in Figure 8, Figure 9 and from the UPGMA tree shown in Figure 10. The AMI profile and the proposed distance measure may, therefore, allow functional distinction between genomes that are evolutionarily comparable but have acquired new biological characteristics.

\section{Discussion}

The observations reported here suggest that the average mutual information analysis of long range genomic structure can yield new insight into the nature of the genome. The data reported here indicate that entire genomic sequences can be analyzed (without the need for multiple alignments) in efforts to gain an understanding of the evolutionary relationship between various species, and among chromosomes within a single species.

As described here, the average mutual information profile of genomic structure reveals a great deal of fine structure in the various sequences available. This structure might possibly have been ignored, except for the fact that so much is highly reproducible among the various chromosomes. Based on the fact that the distances between AMI profiles seem to correlate with evolutionary relationship we speculate that the structure revealed by the average mutual information profiles is closely related to the evolution of various species and their genomes. Finally, as the AMI profile for each sequence is obtained without reference to other sequences there is no need for a multiple sequence alignment when comparing sequences.

\section{Conclusion}

The AMI profile provides a simple, easily computable, species signature. The signature can be used in applications where evolutionary relationships need to be deduced using relatively short fragments of DNA as well as where evolutionary relationships between organisms are to be studied using large genomic sequence. Distances between sets of genomic sequences can be obtained with- 


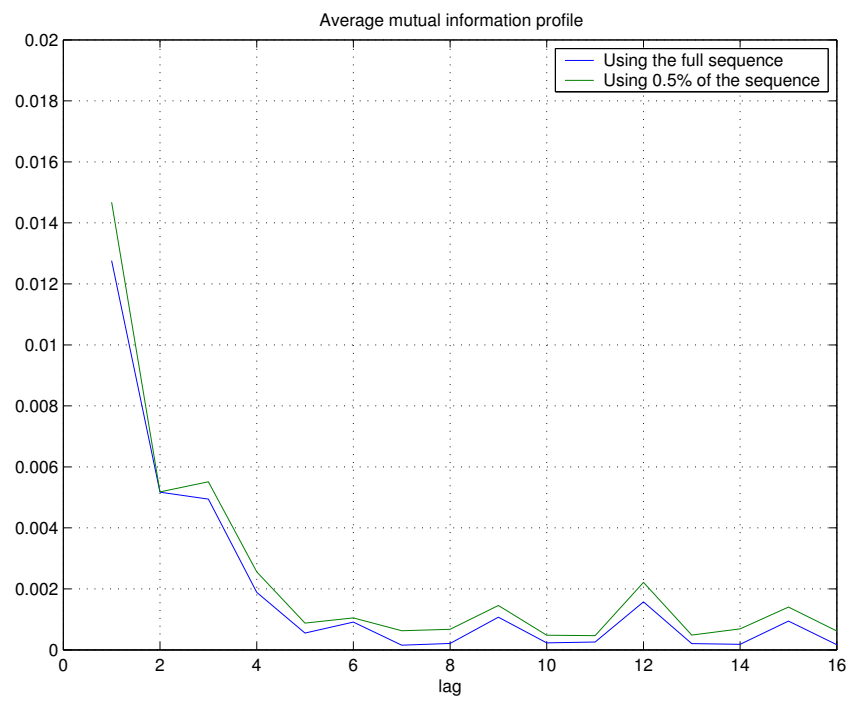

Figure 4

Plot of the first sixteen elements of the average mutual information profile for $E$. coli using the entire sequence and using $0.5 \%$ of the sequence.

out the need for multiple sequence alignment. The profile, and the distance measure associated with it, may also be useful, either by itself or in conjunction with other signatures, to discriminate between fragments of DNA from different species and to identify fragments of genomic DNA with the species of origin.

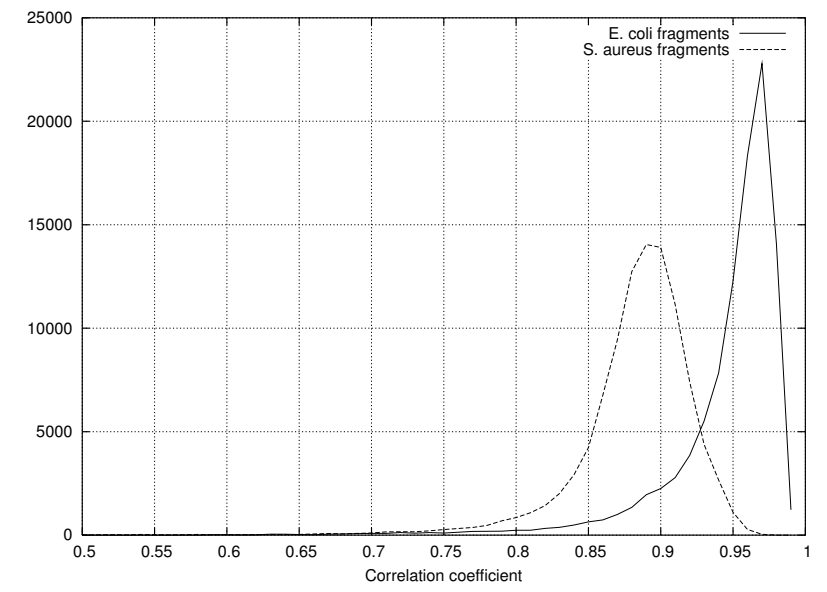

\section{Figure 5}

Plot of the histogram of the correlation between the average mutual information profile of fragments of $E$. coli and $S$. aureus with the average mutual information profile of the entire $E$. coli genome.

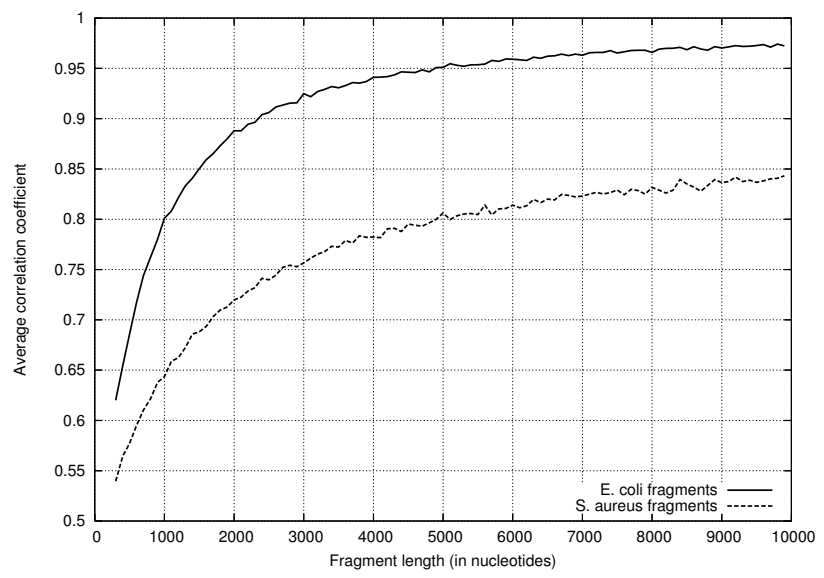

Figure 6

Plot of the average correlation between the average mutual information profile of fragments of $E$. coli and $S$. aureus with the average mutual information profile of the entire $E$. coli genome as a function of fragment length. The average correlation was obtained using 1000 trials with the appropriate fragment length.

\section{Methods}

\section{AMI profile for DNA sequences}

In this work we examine a particular information theoretic measure, average mutual information, as a candidate for species signature. If we have two events $X$ and $Y$ which are independent of each other then the joint probability of occurrence of the two events, $p(X, Y)$ is simply the product of the probability of occurrence of each event, $p(X, Y)$ $=p(X) p(Y)$. Thus, the deviation from unity of the ratio $p(X$, $Y) /[p(X) p(Y)]$, or the deviation from zero of the logarithm of this ratio, can be used as a measure of dependence. If we take $X$ to be the base at some location and $Y$ to be the base at location $k$ downstream from it we can define an average measure of dependence as:

Table I: Labels for chromosomes

\begin{tabular}{|c|c|c|}
\hline & Accession & Chromosome \\
\hline$m \mid 4$ & NT 002582 & M. musculus chromosome 14 \\
\hline$m \mid 7$ & NT 002588 & M. musculus chromosome 17 \\
\hline$M X$ & NT 003030 & M. musculus chromosome $X$ \\
\hline sc3 & NC 001135 & S. cerevisiae chromosome 3 \\
\hline sc5 & NC 001137 & S. cerevisiae chromosome 5 \\
\hline sc9 & NC 001 141 & S. cerevisiae chromosome 9 \\
\hline cel & NC 000965 & C. elegans chromosome I \\
\hline ce2 & NC 000966 & C. elegans chromosome 2 \\
\hline ce3 & NC 000967 & C. elegans chromosome 3 \\
\hline$h \mid 4$ & NT 003140 & H. sapiens Chromosome 14 \\
\hline$h \mid 7$ & NT 002831 & H. sapiens Chromosome 17 \\
\hline$H X$ & NT 001374 & H. sapiens Chromosome $X$ \\
\hline
\end{tabular}

Labels used for the chromosomes of various species in Table 2. 
Table 2: Distance between chromosomes.

\begin{tabular}{ccccccccccccc}
\hline & $s c 9$ & $s c 5$ & $s c 3$ & $m X$ & $m \mid 7$ & $m \mid 4$ & $h X$ & $h \mid 7$ & $h \mid 4$ & $c e 3$ & $c e 2$ & $c e l$ \\
\hline$s c 9$ & 0.000 & 0.018 & 0.017 & 0.512 & 0.485 & 0.513 & 0.549 & 0.539 & 0.536 & 0.377 & 0.373 & 0.355 \\
$s c 5$ & 0.018 & 0.000 & 0.016 & 0.446 & 0.418 & 0.450 & 0.483 & 0.469 & 0.469 & 0.312 & 0.309 & 0.291 \\
$s c 3$ & 0.017 & 0.016 & 0.000 & 0.459 & 0.433 & 0.461 & 0.496 & 0.485 & 0.483 & 0.339 & 0.334 & 0.317 \\
$m X$ & 0.512 & 0.446 & 0.459 & 0.000 & 0.009 & 0.015 & 0.046 & 0.055 & 0.056 & 0.205 & 0.197 & 0.205 \\
$m \mid 7$ & 0.485 & 0.418 & 0.433 & 0.009 & 0.000 & 0.029 & 0.063 & 0.066 & 0.074 & 0.209 & 0.202 & 0.208 \\
$m \mid 4$ & 0.514 & 0.450 & 0.461 & 0.015 & 0.029 & 0.000 & 0.071 & 0.083 & 0.079 & 0.225 & 0.216 & 0.225 \\
$h X$ & 0.549 & 0.483 & 0.496 & 0.046 & 0.063 & 0.071 & 0.000 & 0.003 & 0.002 & 0.197 & 0.186 & 0.199 \\
$h \mid 7$ & 0.539 & 0.469 & 0.485 & 0.055 & 0.066 & 0.083 & 0.003 & 0.000 & 0.004 & 0.189 & 0.179 & 0.189 \\
$h \mid 4$ & 0.536 & 0.469 & 0.483 & 0.056 & 0.074 & 0.079 & 0.002 & 0.004 & 0.000 & 0.188 & 0.178 & 0.189 \\
$c e 3$ & 0.377 & 0.312 & 0.339 & 0.205 & 0.209 & 0.225 & 0.197 & 0.189 & 0.188 & 0.0000 & 0.002 & 0.003 \\
$c e 2$ & 0.373 & 0.309 & 0.334 & 0.197 & 0.202 & 0.216 & 0.186 & 0.179 & 0.178 & 0.002 & 0.000 & 0.004 \\
cel & 0.355 & 0.291 & 0.317 & 0.205 & 0.208 & 0.225 & 0.199 & 0.189 & 0.189 & 0.003 & 0.004 & 0.000
\end{tabular}

Distance between the profiles of Mus musculus, Saccharomyces cerevisiae, $C$. elegans, and Human chromosomes

$$
I_{k}=\sum_{X \in \mathcal{A}} \sum_{Y \in \mathcal{A}} p_{k}(X, Y) \log \frac{p_{k}(X, Y)}{p(X) p(Y)}
$$

where $\mathcal{A}$ is the set of nucleotides $\{A, G, C, T\}$. We have added the subscript $k$ to the joint probability to show that the nucleotides occur $k$ bases apart. By plotting the average mutual information for different values of $k$ we can arrive at a profile for a particular sequence. We refer to this profile as the average mutual information (AMI) profile.

We compute the average mutual information for bases $k$ apart by estimating the probabilities using the relative frequencies of occurrence. Let $n_{k}(X, Y)$ be the number of times two bases $k$ apart take on the values $X$ and $Y$, where $X$ and $Y$ can be $A, C, G$, and $T$. The joint probabilities $p_{k}(X$, $Y$ ) are estimated by

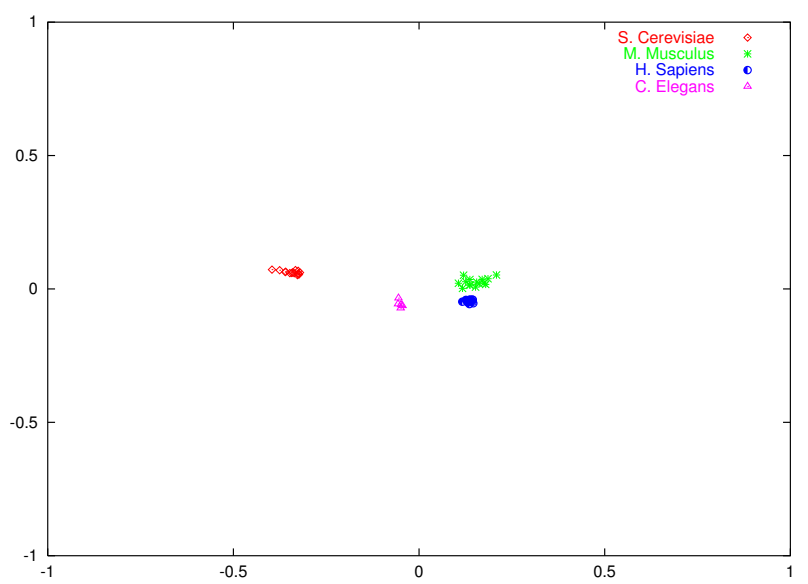

Figure 7

Clustering of all chromosomes from S. cerevisiae, M. musculus, $H$. sapiens and $C$. elegans. The clustering and visualization approach is described in the Methods section.

$$
p_{k}(X, Y)=\frac{n_{k}(X, Y)}{\sum_{I \in \mathcal{A}} \sum_{J \in \mathcal{A}} n_{k}(I, J)}
$$

The marginal probabilities $p(X)$ can similarly be estimated by dividing the total number of times the nucleotide $X$ occurs divided by the total number of bases in the sequence.

\section{The visualization program}

The visualization program was developed in order to visualize the distances between a large number of multidimensional vectors. The particular application was to visualize the distance between AMI profiles of a large number of DNA sequences.

The program requires as its input a list of the sequences $\left\{s_{i}\right\}$ and their distances $\left\{d_{i, j}\right\}$ from each other. The user can also input just the AMI profile of the sequences. The program then calculates the distances. These distances are defined earlier in the paper. Each sequence is treated as a point in a two or three dimensional free space which is operated on by "forces" exerted upon it by the points representing all other sequences.

The points corresponding to the sequences are initially assigned a random locations $l_{i}$ in the unit square or cube and a random velocity $v_{i}$. For each sequence $s_{i}$ a vector "force" $f_{i, j}$ due to all other sequences $s_{j}$ is calculated. The force is defined as

$$
f_{i, j}=\left(d_{i, j}-\hat{d}_{i, j}\right) \mathbf{u}_{j, i}
$$

where $\hat{d}_{i, j}$ is the Euclidean distance between the assigned locations of $s_{i}$ and $s_{j}$ and $\mathbf{u}_{j, i}$ is the unit vector from $s_{j}$ to $s_{i}$. The cumulative force on $s_{i}$ is calculated as 
Table 3: Labels for HIV subtypes

\begin{tabular}{|c|c|c|}
\hline & Acc. No. & Description \\
\hline$a l$ & AF004885 & HIV-I isolate from Kenya (Subtype A) \\
\hline$a 2$ & AF06967I & HIV-I isolate from Sweden, (Subtype A) \\
\hline$a 3$ & U5II90 & HIV-I, isolate from Uganda (Subtype A) \\
\hline$a 4$ & AF069672 & HIV-I isolate from Sweden (Subtype A) \\
\hline$a 5$ & AFI0777I & HIV-I isolate from Sweden (Subtype A) \\
\hline$a 6$ & M62320 & HIV-I Ugandan isolate (Subtype A) \\
\hline$a 7$ & AF069670 & HIV-I isolate from Somalia (Subtype A) \\
\hline$b l$ & AF042I0I & HIV-I isolate from Australia (Subtype B) \\
\hline$b 2$ & U37270 & HIV-I isolate from Australia (Subtype B) \\
\hline b3 & U43096 & HIV-I isolate from Germany (Subtype B) \\
\hline b4 & $\cup 43|4|$ & HIV-I isolate from Germany (Subtype B) \\
\hline$b 5$ & AJ006287 & HIV-I isolate from Spain (Subtype B) \\
\hline$b 6$ & AFI46728 & HIV-I from Australia (Subtype B) \\
\hline b7 & U7II82 & HIV-I isolate from China (Subtype B) \\
\hline cl & AFII0960 & HIV-I isolate from Botswana (Subtype C) \\
\hline$c 2$ & AFII 0959 & HIV-I isolate from Botswana (Subtype C) \\
\hline$c 3$ & U52953 & HIV-I isolate from Brazil (Subtype C) \\
\hline$c 4$ & AF067I57 & HIV-I isolate from India (Subtype C) \\
\hline$c 5$ & AF067I55 & HIV-I isolate 21068 from India (Subtype C) \\
\hline c6 & U46016 & HIV-I Human immunodeficiency virus type I (subtype C) \\
\hline c7 & $\mathrm{AB} 023804$ & HIV-I Human immunodeficiency virus type I (subtype C) \\
\hline
\end{tabular}

List of accession numbers, descriptions, and labels of HIV I sequences used to examine distances between subtypes.

$$
f_{i}=\sum_{j} f_{i, j}
$$

This force is calculated for each of the sequences. The vector velocities of the sequences are then updated by displacing them by an amount proportional to the vector force on them. The update equation for sequence $s_{i}$ at time $n+1$ is given by

$$
v_{i}^{(n+1)}=\alpha\left(v_{i}^{(n)}+\beta f_{i}^{(n)}\right)
$$

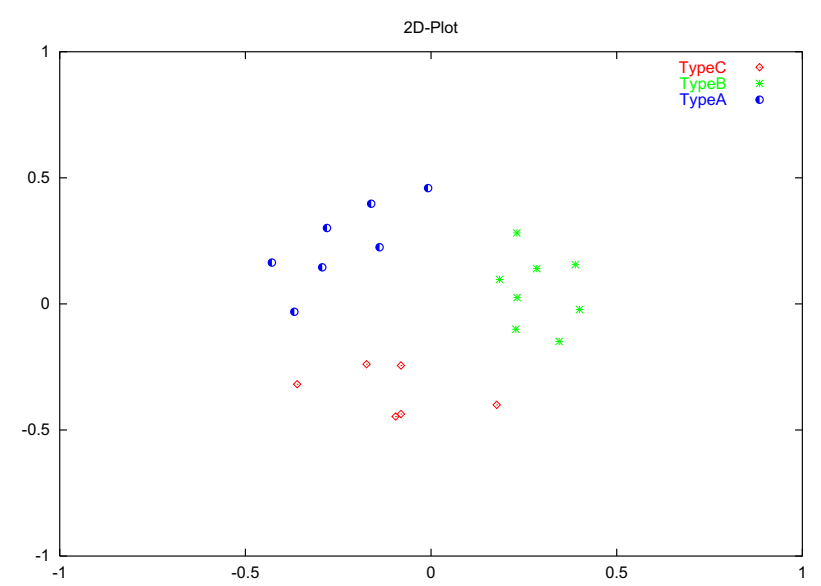

Figure 8

Clustering of HIV-I subtypes based on the distance between their respective $\mathrm{AMI}$ profiles. where the superscripts denote the iteration counter. The locations of the sequences are then updated by displacing them by an amount proportional to the vector velocities.

$$
l_{i}^{(n+1)}=l_{i}^{(n)}+\gamma v_{i}^{(n+1)}
$$

The constants $\alpha, \beta$, and $\gamma$ were experimentally determined to provide a good tradeoff between rate of convergence and jitter. A larger value for these constant will permit a faster convergence with considerable jitter around the final configuration, and vice versa. We picked the con-

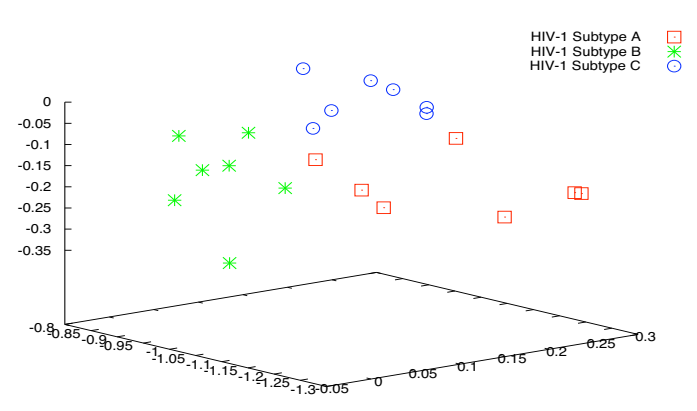

\section{Figure 9}

Clustering of HIV-I subtypes evidenced by three coefficients of the singular valued decomposition of the AMI profiles. 


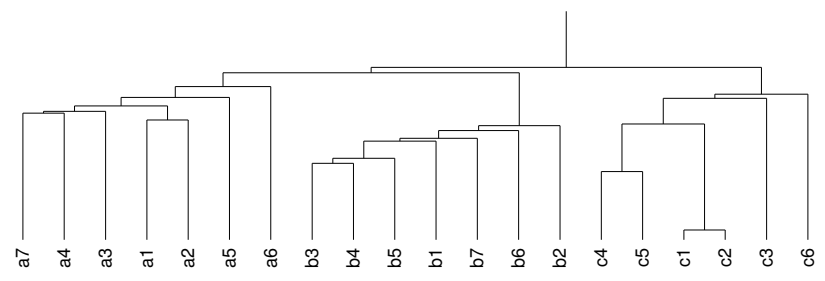

Figure 10

UPGMA tree for subtypes of the HIV-I virus. The distances used to construct the UPGMA tree were obtained from their respective AMI profiles. The labels used here are defined in Table 3.

stants to be $0.7,0.1$, and 0.1 . After the locations are updated the process is repeated until the sequences have stabilized in their locations. Our observation is that it requires about fifty iterations for the configuration to stabilize. Keeping in mind that there are multiple stable configurations and to prevent the system from settling into a local minimum, we randomly perturb the configuration every 50 updates. The size of the random perturbations is uniformly distributed in the interval $[-.5, .5]$ and is multiplied by $0.95^{m}$ where $m$ is the number of random perturbations applied to this point. The $2 \mathrm{D}$ version of the program is available for use at [43].

\section{Authors' contributions}

KS conceived the study, and together with $\mathrm{MB}$ designed and tested the algorithm. SS provided the biological insight. All authors read and approved the final manuscript.

\section{Acknowledgements}

The authors thank Professor David Brooks, Professor Myron Brakke, and Professor H.H. Otu for their encouragement and very constructive critiques. KS thanks NIH for support under grant K25AI068I5I.

\section{References}

I. Gates M: A Simple Way to Look at DNA. J Theor Biol 1986, I 19:319-328.

2. Voss R: Evolution of long-range Fractal Correlations and I/f Noise in DNA Base Sequences. Phys Rev Letters 1992, 68(25):3805-3808.

3. Peng C, Buldyrev S, Goldberger A, Havlin S, Sciortino F, Simons M, Stanley H: Long Range Correlations in Nucleotide Sequences. Nature 1992, 356:168-170.

4. Buldyrev S, Goldberger A, Havlin A, Peng C, Simons M, Stanley H: Generalized Levy-Walk Model for DNA Nucleotide Sequences. Phys Rev E 1992, 47(6):45 I 4-4523.

5. Allegrini P, Barbi M, Grigolini P, West B: Dynamical Walk Model for DNA Sequences. Phys Rev E 1995, 52(5):528I-5296.

6. Gatlin L: Information Theory and the Living System New York: Columbia University Press; 1972.

7. Roman-Roldan R, Bernaolo-Galvan P, Oliver J: Application of Information Theory to DNA Sequence Analysis: A Review. Pattern Recognition 1996, 29(7): I 187-I194.

8. Gold TSGSL, Ehrefeucht A: Information Content of Binding Sites on Nucleotide Sequences. J Mol Biol 1986, I 88:4I5-43I.

9. Schneider T, Stephens R: Sequence Logos: A New Way to Display Consensus Sequences. Nucleic Acid Res 1990, 18:6097-6100.
10. Schneider T, Mastronade D: Fast Multiple Alignment of Ungapped DNA Sequences Using Information Theory and a Relaxation Method. Discrete Applied Mathematics 1996, 7I:259-268.

1I. Giraud B, Lapedes A, Liu L: Analysis of Correlations Between Sites in Models of Protein Sequences. Phys Rev E 1998, 58(5):63| 2-6322

12. Herzel H, Grosse I: Correlations in DNA Sequences: The Role of Protein Coding Segments. Phys Rev E 1997, 55:800-810.

13. Korber B, Farber R, Wolpert D, Lapedes A: Covariation of Mutations in the V3 Loop of Human Immunodeficiency Virus Type I Envelope Protein: An Information Theoretic Analysis. Proc Natl Acad Sci 1993, 90:7176-7I80.

14. Luo L, Lee W: Statistical Correlation of Nucleotides in a DNA Sequence. Phys Rev E 1998, 58:86|-87|

15. Roman-Roldan R, Bernaolo-Galvan P, Oliver J: Sequence Compositional Complexity of DNA through an Entropic Segmentation Method. Phys Rev Letters 1999, 80(6): I 344-I347.

16. Bernaolo-Galvan P, Oliver J, Ramon-Roldan R: Decomposition of DNA Sequence Complexity. Phys Rev Letters 1999:3336-3339.

17. Cardon SKL: Computational DNA Sequence Analysis. Annu Rev Microbiol 1994, 48:619-654.

18. Karlin S, Mocarski E, Schachtel G: Molecular Evolution of Herpesviruses: Genomic and Protein Sequence Comparisons. J Virol 1994, 68(3): 1886-1902.

19. Karlin S: Statistical Significance of Sequence Patterns in Proteins. Curr Opin Struct Biol 1995, 5(3):360-37I.

20. Blaisdell B, Campbell A, Karlin S: Similarities and Dissimilarities of Phage Genomes. Proc Natl Acad Sci USA 1996, 93(I 2):5854-5859.

21. Karlin S, Mrazek J, Campbell A: Compositional Biases of Bacterial Genomes and Evolutionary Implications. J Bacteriol I997, I79(12):3899-3913.

22. Brendel V, Beckmann J, Trifonov E: Linguistics of Nucleotide Sequences: Morphology and Comparison of Vocabularies. $J$ Biomol Struct Dyn 1986, 4:I I-2I.

23. Beckmann J, Brendel V, Trifonov E: Intervening Sequences Exhibit Distinct Vocabulary. J Biomol Struct Dyn 1986, 4(3):39I-400.

24. E EB, Pizzi E, Giudice PD, Frontali C: Pentamer Vocabularies Characterizing Introns and Intron-like Intergenic Tracts from Caenorhabditis elegans and Drosophila melanogaster. Gene 2003, 304:183-192.

25. Dufraigne C, Fertil B, Lespinats S, Giron A, Deschavanne P: Detection and Characterization of Horizontal Transfers in Prokaryotes Using Genomic Signature. Nuc Acids Res 2005, 33:e6.

26. Tyson G, Chapman J, Hugenholtz P, Allen E, Ram R, Richardson P, Solovyev V, Rubin E, Rokhsar D, Banfield J: Community Structure and Metabolism Through Reconstruction of Microbial Genomes from the Environment. Nature 2004, 428(6978):37-43.

27. Venter J, Remington K, Heidelberg J, Halpern A, Rusch D, Eisen J, Wu D, Paulsen I, Nelson K, Nelson W, Fouts D, Levy S, Knap A, Lomas M, Nealson K, White O, Peterson J, Hoffman J, Parsons R, Baden-Tillson H, Pfannkoch C, Rogers Y, Smith H: Environmental Genome Shotgun Sequencing of the Sargasso Sea. Science 2004, 304(5667):66-74.

28. Karlin S, Burge C: Dinucleotide Relative Abundance Extremes: A Genomic Signature. Trends Genet 1995, I I:283-290.

29. Deschavanne P, Giron A, Vilain J, Fagot G, Fertil B: Genomic Signature: Characterization and Classification of Species Assessed by Chaos Game Representation of Sequences. Mol Biol Evol 2000, 16:1391-1399.

30. Sandberg R, Winberg G, Branden Cl, Kaske A, Ernberg I, Coster J: Capturing Whole-Genome Characteristics in Short Sequences Using a Naive Bayesian Classifier. Genome Res 200I, I I(8): I404-I409.

31. Shannon C: A Mathematical Theory of Communication. Bell Syst Tech J 1948, 27:379-423. 623-656

32. Hofacker I, Fekete M, Stadler P: Secondary structure prediction for aligned RNA sequences. Journal of Molecular Biology 2002, 319:1059-1066.

33. Lindgreen S, Gardner P, Krogh A: Meauring covariation in RNA alignments: physical realism improves information measure. Bioinformatics 2006, 22:2988-2995. 
34. Grosse I, Herzel H, Buldyrev S, Stanley H: Species Independence of Mutual Information in Coding and Noncoding Regions. Phys Rev E 2000, 6 I (5):5624-5629.

35. Slonim N, Atwal G, Tkacik G, Blalek W: Informantion-based clustering. Proceedings of the National Academy of Sciences 2005, 102:18297-18302.

36. Slonim N, Elemento $O$, Tavazole $S$ : Ab initio genotype-phenotype association reveals intrinsic modularity in genetic networks. Molecular Systems Biology 2006:2.

37. Berryman M, Allison A, Abbott D: Mutual Information for Examining Correlations in DNA. Fluctuation and Noise Letters 2004, 4(2):L237-L246.

38. Holste D, Beirer S, Schieg P, Grosse I, Herzel H: Repeats and Correlations in Human DNA Sequences. Phys Rev E 2003, 67:061913 I-061913 7.

39. Dehnert M, Helm W, Hutt MT: Information theory reveals large scale synchronisation of statistical correlations in eukaryote genomes. Gene 2005, 345:8I-90.

40. Dehnert M, Helm W, Hutt MT: Informational structure of two closely related eukaryote genomes. Physical Review E 2006, 74:021913-1-021913-9.

41. Hummel J, Keshvari N, weckwerth W, Selbig J: Species-specific analysis ofprotein sequences using mutual information. $B M C$ Bioinformatics 2005:6.

42. Martin L, Gloor G, Dunn S, Wahl L: Using Information Theory to Search for Co-evolving Residues in Proteins. Bioinformatics 2005, 21:4| |6-4|24.

43. Average Mutual Information Distance Plotter [http://
[ sensin.unl.edu/bioinformatics]

Publish with Bio Med Central and every scientist can read your work free of charge

"BioMed Central will be the most significant development for disseminating the results of biomedical research in our lifetime. "

Sir Paul Nurse, Cancer Research UK

Your research papers will be:

- available free of charge to the entire biomedical community

- peer reviewed and published immediately upon acceptance

- cited in PubMed and archived on PubMed Central

- yours - you keep the copyright 